\title{
Describing Atomic-Level Fluxional Behavior in Nanoparticles
}

\author{
Ramon Manzorro ${ }^{1}$, Joshua Vincent ${ }^{1}$, David Matteson ${ }^{2}$, Yuchen $\mathrm{Xu}^{2}$ and Peter Crozier ${ }^{3}$ \\ ${ }^{1}$ Arizona State University, United States, ${ }^{2}$ Cornell University, United States, ${ }^{3}$ Arizona State University, \\ Tempe, Arizona, United States
}

In situ electron microscopy in gas and liquid environments provides critical information on a wide range of materials phenomena such as nucleation and growth, phase transformations, and diffusion processes. Timeresolved in situ TEM observations can provide detailed atomic-level information on kinetic pathways that operate when the material is in its working state, offering the ability to directly observe dynamic changes in the material in real time [1-4]. For many processes, such as catalytic reactions or phase changes, materials may show significant atomic level structural dynamics or so-called fluxional behavior. To characterize such behavior with in situ microscopy, one would ideally like to identify the location of every atom as a function of time. Hardware considerations, such as detector readout rates (currently $\sim 10^{3}$ frames per second on commercially available systems) define the achievable temporal resolution. However, in practice, signal-tonoise considerations often impose an inverse relationship between the achievable temporal and spatial resolution [5, 6]. Here we discuss different types of fluxional behavior and describe approaches to extract spatio-temporal information. We also consider frameworks that may be developed to provide quantitative descriptions of such dynamics.

Fluxional behavior involving lattice distortion and strain can be analyzed by fitting functions such as Gaussians to the images to determine the location and intensity of each atomic column. We have performed time dependent measurements of the cation sublattice in $\mathrm{CeO}_{2}$ to identify surface sites for oxygen vacancy creation and annihilation. At room temperature, the $10-20 \mathrm{pm}$ fluctuation in the Ce cation position is slow enough to be tracked at 40 frames per second with adequate precision $[3,5]$. If the time scale of the fluxional behavior is much shorter than the detector exposure time, or if low signal levels require frame averaging, images of atomic columns will become blurred (possibly in an anisotropic fashion) and it is no longer possible to determine the column position as a function of time. However, by measuring the column width, local fluxional strain can be determined, and this has been exploited to obtain information about changes in the oxygen exchange rate on $\mathrm{Pt} / \mathrm{CeO}_{2}$ catalysts performing $\mathrm{CO}$ oxidation [7].

In more pronounced structural dynamics, such as surface migration, kinetic information can be determined provided the dynamics manifests in terms of a series of metastable states which are mostly stationary during the frame exposure time. Under such conditions, each image in the series, corresponding to a well-defined state, will show well-defined atomic columns and simple functions can still be employed to quantify the image intensity. To mitigate signal-to-noise issues, we have found techniques such as blob detection to be more robust than Gaussian peak fitting for image quantification. We have characterized surface structural dynamics at 2.5 ms time resolution on a $\mathrm{CeO}_{2}$ nanoparticle using intense electron irradiation to drive oxygen transport and surface vacancy creation processes. Oxygen vacancies are constantly created at the surface and annihilated by oxygen transport from the bulk. When the oxygen coordination around a surface Ce cation is low, surface cation diffusion is likely, leading to changes in the surface configuration or state. The entire data set ( 9000 frames) can be described in terms of 12 distinct metastable surface states consisting in different atomic rearrangement taking place mostly at the surface of the nanoparticle. Figure 1a and b show typical examples of infrequent and frequently occurring states respectively. Not surprisingly, the infrequent state is associated with an unstable adatom structural configuration. Transition matrices can be constructed to quantify the kinetics of the $12^{2}=144$ different transition pathways between the 12 states. Hidden Markov models may potentially establish stronger links to the underlying kinetics and statistical mechanics. Such approaches can be used to 
directly link structure, structural dynamics and kinetics, providing a framework to give a quantitative description of the fluxional behavior.

The cation dynamics observed in Figure 1 are driven by oxygen activity (surface vacancies and bulk transport). At some periods in time, more extreme fluxional behavior in the $\mathrm{CeO}_{2}$ can result in the loss of atomic column contrast in the image. Figure 1c shows another point in time where a complete loss of atomic column contrast occurs on one half of a (111) plane that is labelled Row 4. This is likely related to crystallographic shearing due to the very high local concentration of oxygen vacancies at this point in time near this cation plane. Different approaches must be developed to describe such behavior, using for example, Miller plane spacing, the length of the sheared section, as well long-range order parameters derived from Patterson type approaches [8].
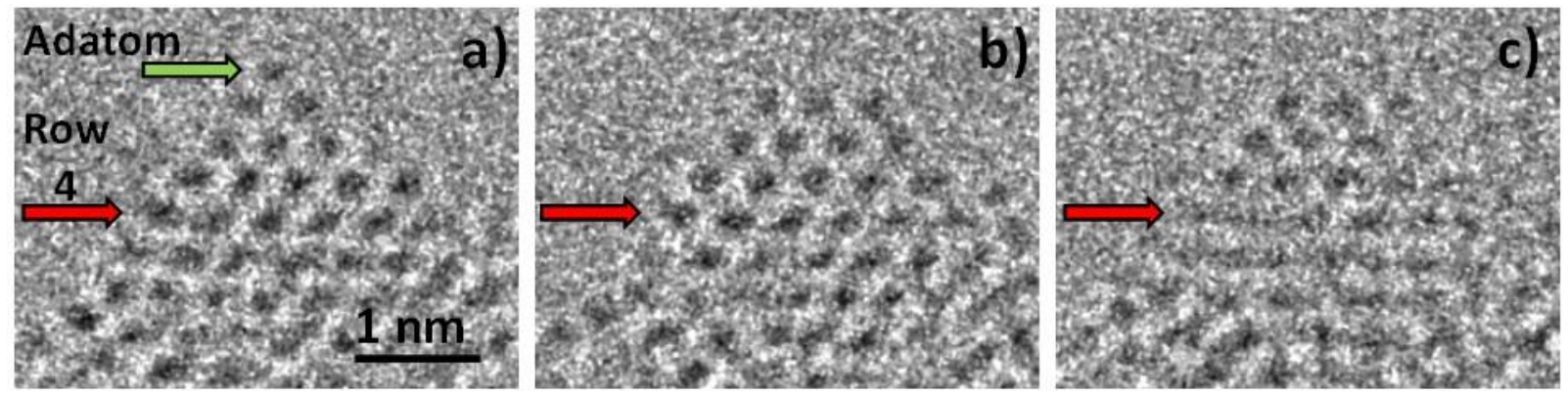

Figure 1. Figure 1. Structural states observed at different points in time for a $\mathrm{CeO} 2$ nanoparticles $(2.5 \mathrm{~ms}$ frame time, 2 frame average to enhance signal-to-noise). a) Rare state showing adatom column (green arrow). b) Common state showing complete terrace of 3 columns. c) Loss of periodicity on half of row 4 (red arrow) due to onset of crystallographic shear.

\section{References}

1. Miller, B.K. and P.A. Crozier, ACS Catalysis 2021. 11: p. 1456-1463.

2. Li, Y., et al., Nature Communications, 2021. 12(1): p. 914.

3. Lawrence, E.L., et al., ACS Nano, 2021. https://doi.org/10.1021/acsnano.0c07584

4. Vincent, J.L., et al., Ultramicroscopy, 2020. 218: p. 113080.

5. Levin, B.D.A., E.L. Lawrence, and P.A. Crozier, Ultramicroscopy, 2020. 213: p. 112978.

6. Lawrence, E.L., et al., Microscopy and Microanalysis, 2020. 26(1): p. 86-94.

7. Vincent, J.L. and P.A. Crozier, (in preparation), 2021.

8. Haiber, D.M., et al., Chemistry of Materials, 2021. 33(1): p. 195-204.

9. We gratefully acknowledge support of NSF grants CBET-1604971, DMR-1308085, OAC-1940263, CCF1934985, OAC-1940276, and the use of (S)TEM at Eyring Materials Center at Arizona State University is gratefully acknowledged. 